In this paper, the complete model (kinematic and dynamic) of the quadrotor helicopter is presented, based on the Newton-Euler approach with analysis of gravity, aerodynamics, damping and thrust. This model is developing by $[2,4,25]$. The main objective of this article is the synthesis of stabilizing control laws in terms of translation and orientation for the X4. It's a vehicle has the difficulty of the control because its complex, non-linear dynamics, multivariable, of coupled nature, particularly in its operation and sensitivity to the external disturbance. The PD-CTC controller is proposed to solve this problem (control). The control strategy is based on the decomposition of the original system into two subsystems: the first concerns the position control while the second is to control orientation and the velocity.

The quadrotor is composed of four rotors in a cross configuration, this cross structure is quite thin and light, which will guarantee robustness by ensuring the mechanical links for the motors, for reasons to simplifying the model; the axes of propellers are fixed in parallel and each propeller is combined with a motor, these configurations will help us to maneuver the quadrotor just by varying the speed of propellers.

The most important thing in this configuration that the pair front-rear propellers rotate counter clockwise while the left-right one rotates clockwise that removes the need for a tail rotor (needed in the classic helicopter), in the hovering condition, all the propellers must have the same speed. In Figure 1 a block diagram of the quadrotor structure is presented in black, the fixed-body B-frame is shown in green and in blue is represented the angular speed of the propellers.in addition to the name of the velocity variable, for each propeller, two arrows are drawn: the curved one represents the direction of rotation and the second represents the velocity. In order to perform a stationary flight(hovering), the propellers must rotate at the same speed for countering the acceleration due to the gravity, as depicted in Figure 1.

The quadrotor is equipped just with four rotors so for controlling the engine, we have to choose a number of freedoms that correspond to the number of actuators so for our case we were choose 3 dgrees of freedom which are roll angle, pitch angle, yaw angle. The quadrotor can perform four basic movements which allow this engine to reach a certain altitude(targets); the principal movements are described:

Throttle (U1): this command is provided by increasing (or decreasing) all the propeller speeds by the same amounts $\Delta_{A}$ like in Figure 2, where $\Omega_{H}$ is the speed of propellers.it leads to a vertical force which raises or lowers the quadrotor, the amount $\Delta_{A}$ must be too small because the model would eventually influenced by strong non linearities or saturations.

Roll (U2): this command is provided by increasing (or decreasing) the left propeller speed and inverse operation for the right one. It deals with the torque around the $x_{B}$ axis. The amounts $\Delta_{A}$ and $\Delta_{B}$ in the Figure 3 must be equals for the same reasons mentioned above and also to maintain the vertical thrust unchanged.

Pitch (U3): this command is similar to the roll and is provided by increasing (or decreasing) the rear propeller speed and inverse operation for the front one.it leads to a torque around the $y_{B}$ axis. The positive amounts $\Delta_{A}$ and $\Delta_{B}$ are chosen to maintain the vertical thrust unchanged and can't be too large as in Figure 4 .

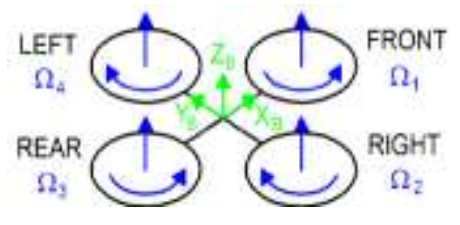

Figure 1. Simplified quadrotor

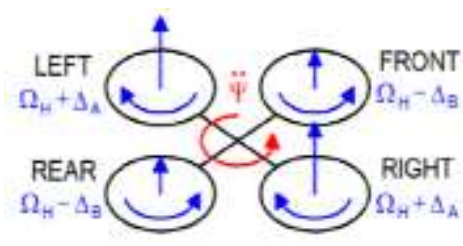

Figure 3. Roll movement

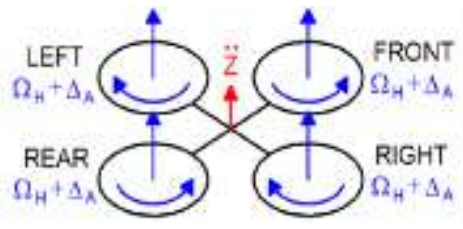

Figure 2. Throttle movement.

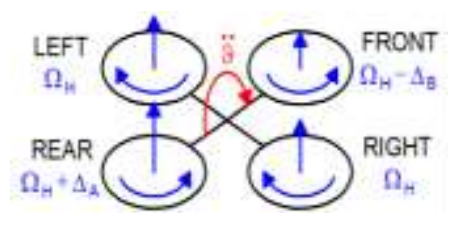

Figure 4. Pitch movement

Yaw (U4): this command is provided by increasing (or decreasing) the front-rear propellers speed and the inverse operation for the left-right pair.it leads to a torque with respect to the $z_{B}$ axis, hence the front- 
rear couple rotate counter clockwise and the left-right one rotate clockwise. The same technic for $\Delta_{A}$ and $\Delta_{B}$ that are chosen to maintain the vertical thrust unchanged and they can't be too large as shown in the Figure 5.

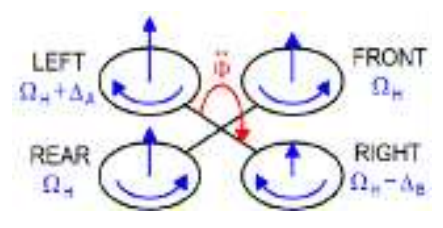

Figure 5. Yaw movement

\section{QUADROTOR MODELING}

In this section, we will describe the reference frames and coordinate systems which will served to extract the positions and orientations of quadrotor.A transformation matrix between these coordinate systems is developed for the following reasons:

- Newton's equations of motion are given in the body coordinate frame.

- Aerodynamics forces and torques are applied in the earth frame.

Unlike GPS which is used to measure position, ground speed and course angle with respect to the earth frame,the on-board sensors like accelerometers and gyroscopes are used for gathering information in the body frame. for trajectory planification missions, all the collected information have to been transformed in the inertial frame. To switch from one coordinate frame to another, two basic operations are performed: rotations and translations.

As described in [2-4] the details of dynamic model of the quadrotor will not be discussed in this paper again.for reasons cited previously, we will consider two frames; earth frame and a body frame whose origin is in the center of mass of our engine, see Figure 1. The attitude vector of the quadrotor is given by the roll, pitch and yaw angles, forming the vector $\Omega^{T}=(\phi, \theta, \psi)$ however the position of the vehicle in the inertial frame is given by the position vector $r^{T}=(x, y, z)$. Conservation of momentum and angular momentum laws are used to extract the dynamic model of the quadrotor, considering the applied forces and torques into account $[4,26]$. The thrust force generated by rotor $i, i=1,2,3,4$ is $F_{i}=b * w_{i}^{2}$ with the thrust factor $b$ and the rotor speed $w_{i}$, and the law of conservation of momentum yields;

$$
\dot{r}=g \cdot\left(\begin{array}{l}
0 \\
0 \\
1
\end{array}\right)-R(\Omega) \cdot \frac{b}{m} \sum_{i=1}^{4} w_{i}^{2}\left(\begin{array}{l}
0 \\
0 \\
1
\end{array}\right)
$$

Herein, $R(\Omega)$ is a suitable rotation matrix and mis the mass of quadrotor. With the inertia matrix $I$ (a pure diagonal matrix with the inertias $I_{x}, I_{y}$ and $I_{z}$ on the main diagonal), the rotor inertia $I_{R}$, the vector $M$ of the torque applied to the vehicle' s body and the vector $M_{G}$ of the gyroscopic torques of the rotors, the law of conservation of angular momentum yields:

$$
I \dot{\Omega}=-(\dot{\Omega} \times I \dot{\Omega})-M_{G}+M
$$

The vector $M$ is defined as;

$$
M=\left(\begin{array}{c}
L b\left(w_{2}^{2}-w_{4}^{2}\right) \\
L b\left(w_{1}^{2}-w_{3}^{2}\right) \\
d\left(w_{1}^{2}-w_{2}^{2}+w_{3}^{2}-w_{4}^{2}\right)
\end{array}\right)
$$

With the drag coefficient $d$ and the length $L$ of the lever. The gyroscopic torques caused by rotations of the vehicle with rotating rotors are:

$$
M_{G}=I_{R}\left(\dot{\Omega} \times\left(\begin{array}{l}
0 \\
0 \\
1
\end{array}\right)\right) \cdot\left(w_{1}-w_{2}+w_{3}-w_{4}\right)
$$


The four rotational velocities $w_{i}$ of the rotors are the real input variables of the vehicle, but for a simplification of the model, the following substitute input variables are defined:

$$
\left\{\begin{array}{l}
u_{1}=b\left(w_{1}^{2}+w_{2}^{2}+w_{3}^{2}+w_{4}^{2}\right) \\
u_{2}=b\left(w_{2}^{2}-w_{4}^{2}\right) \\
u_{3}=b\left(w_{1}^{2}-w_{3}^{2}\right) \\
u_{4}=d\left(w_{1}^{2}-w_{2}^{2}+w_{3}^{2}-w_{4}^{2}\right)
\end{array}\right.
$$

Defining: $u^{T}=\left(u_{1}, u_{2}, u_{3}, u_{4}\right)$ and $\left(w_{1}-w_{2}+w_{3}-w_{4}\right)=g(u)$ and introducing the vector of state variables: $x^{T}=(\dot{x}, \dot{y}, \dot{z}, \phi, \theta, \psi, \dot{\phi}, \dot{\theta}, \dot{\psi})$ evaluation of (1) until (5) yields the following state variable model:

$$
x^{\square}=\left\{\begin{array}{l}
-\left(\cos x_{4} \sin x_{5} \cos x_{6}+\sin x_{4} \sin x_{6}\right) \cdot \frac{u_{1}}{m} \\
-\left(\cos x_{4} \sin x_{5} \sin x_{6}-\sin x_{4} \cos x_{6}\right) \cdot \frac{u u_{1}}{m} \\
g-\left(\cos x_{4} \cos x_{5}\right) \cdot \frac{u_{1}}{m} \\
x_{7} \\
x_{8} \\
x_{8} x_{9} I_{1}-\frac{I_{R}}{I_{x}} x_{8} g(u)+\frac{L}{I_{x}} u_{2} \\
x_{7} x_{9} I_{2}+\frac{I_{R}}{I_{y}} x_{7} g(u)+\frac{L}{I_{y}} u_{3} \\
x_{7} x_{8} I_{3}+\frac{1}{I_{z}} u_{4}
\end{array}\right\}
$$

Herein, we use the abbreviations $I_{1}=\frac{\left(I_{y}-I_{z}\right)}{I_{x}}, I_{2}=\frac{\left(I_{z}-I_{x}\right)}{I_{y}}$ and $I_{3}=\frac{\left(I_{x}-I_{y}\right)}{I_{z}}$, in order to study the dynamic behavior of the quadrotor a 3DOF model is designed in Matlab and the open loop response is shown in the Figure 6.

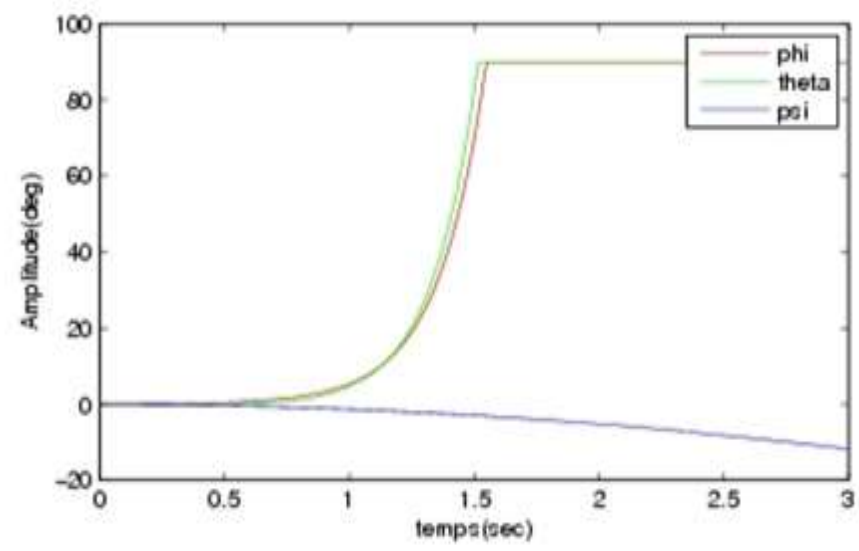

Figure 6. Open loop response of the quadrotor

From the Figure 6 we see that the system is unstable and diverges to the saturation that is introduced to counterbalance the problem of gimbal lock (the angles orientation must be limited to 90degrees in order to 
keep the model operational). These equations are used to well describing the aerodynamic vehicle study, because of the possibility of formulating them from trimmed aerodynamic data and simple autopilot designs. Nevertheless, they give a realistic picture of the translational and rotational dynamics unless large angles and cross-coupling effects dominate the simulations.

\section{CONTROL INVESTIGATION}

The controller of vehicle has the task of stabilization of desired orientation (attitude). In order to achieve and maintain a desired angle orientation. In this section we will discuss the ability of PD-CTC controller to stabilize the attitude of quadrotor, first we need to decompose the state space vector and take just the three angles of orientation $(\phi, \theta, \psi)$. The PD-CTC controller is applied to a nonlinear systems so for this reason is not to linearize our system, we choose to take some assumptions that can help us to easily manipulate the orientation and applied the controller, first, the gyroscopic effect is neglected because the rotor inertia is very small with relative to the quadrotor inertia, the structure of quadrotor is fully symmetric and the inertia matrix is diagonal because the coupled inertia between axis is very low with relative to the inertia of each axis, as shown in Figure 7.

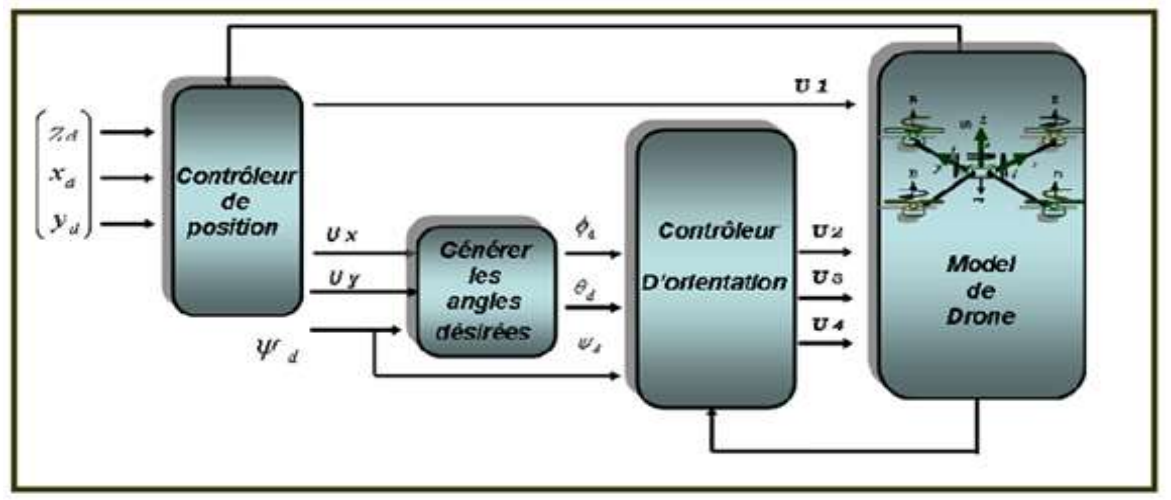

Figure 7. PD-CTC control of the quadrotor

The computed torque algorithm to develop the control allowing the system to follow the desired trajectory. We consider the tracking error:

$e_{z}=z-z_{r}$

$\mathrm{V}_{\mathrm{r}}:$ is the desired velocity.

Its derivative is computed as:

$\dot{e}_{z}=\dot{z}-\dot{z}_{r}$

The nonlinear feedback control law that guarantees tracking of desired trajectory. Selecting Proportional plus derivative (PD) feedback for U (t) results in the PD-computed torque controller [7], the final control is:

$U_{1}=\frac{\left(\ddot{z}_{r}-K_{Z 1} e_{z}-K_{Z 2} \dot{e}_{z}-g\right) m}{\cos x_{4} \cos x_{5}}$

and the resulting linear error dynamics are:

$\left(\ddot{z}_{\text {ref }}-K_{z 1} e_{z}-K_{z 2} \dot{e}_{z}\right)=0$

According to linear system theory, convergence of the tracking error to zero is guaranteed: 


$$
\left\{\begin{array}{l}
u_{2}=\frac{I_{x}}{L}\left(\ddot{x}_{4 r e f}-K_{4_{1}} e_{4_{7}}-K_{4_{2}} \dot{e}_{x_{4}}-x_{8} x_{9} I_{1}-\frac{I_{R}}{I_{x}} g(u) x_{8}\right) \\
u_{3}=\frac{I_{y}}{L}\left(\ddot{x}_{5_{r e f}}-K_{5_{1}} e_{x_{5}}-K_{5_{2}} \dot{e}_{x_{5}}-x_{7} x_{9} I_{2}-\frac{I_{R}}{I_{y}} g(u) x_{7}\right) \\
u_{4}=I_{z}\left(\ddot{x}_{6 r e f}-K_{6_{1}} e_{x_{6}}-K_{6_{2}} \dot{e}_{x_{6}}-x_{7} x_{8} I_{3}\right) \\
x_{5}=\arcsin \left(\frac{\left.\left[-\frac{m}{u_{1}} \ddot{x}_{r e f}-K_{x_{1}} e_{z}-K_{x 2} \dot{e}_{x}\right)+\sin x_{4} \cos x_{6}\right]}{\cos x_{4} \sin x_{6}}\right) \\
x_{4}=\arccos \left(\frac{\left[-\frac{m}{u_{1}}\left(\ddot{y}_{r e f}-K_{y 1} e_{y}-K_{y_{2}} \dot{e}_{y}\right)-\sin x_{4} \sin x_{6}\right]}{\cos x_{6} \sin x_{5}}\right)
\end{array}\right.
$$

where $\mathrm{K}_{\mathrm{X} 1}, \mathrm{~K}_{\mathrm{X} 2}, \mathrm{~K}_{\mathrm{Y} 1}, \mathrm{~K}_{\mathrm{Y} 2}, \mathrm{~K}_{\mathrm{Z} 1}$ and $\mathrm{K}_{\mathrm{Z} 2}$ are the coefficients ensuring stability and positive constants. Where: $l$ is the arm length of quadrotor, $\left(U_{2}, U_{3}, U_{4}\right)$ are controllers, $\left(I_{x x}, I_{y y}, I_{z z}\right)$ are the moments of inertia of quadrotor and $(\ddot{\phi}, \ddot{\theta}, \ddot{\psi})$ are angular accelerations.

The four rotors are coupled two by two in order to counterbalance the gyroscopic effect so from the Figure 7 we can see that there are two pwm motors which began positive and attenuate until they vanish and the two others began negative and arise until they vanish this indicate that these two motors must rotate in opposite with the two others for the reason mentioned above.

\section{SIMULATION RESULTS}

To evaluate the designed control system, repetitive simulation tests were performed via numerical simulation. The control system was simulated using the variable step Runge-Kutta integrator in MATLAB. The airship is tested in simulation in order to validate motion planning algorithm considering the proposed Computed Torque controller.

Figures 8 and 10 show the tracking of desired trajectory by the simulated one and the evolution of the X4-flyer and its stabilisation in 3D displacement for the the straight circle and conical trajectories respectively. The simulation results of PD-CTC control with accurate parameters and without external disturbances and to confirm the robustness of proposed trajectory tracking control method. As illustrated in the above figures, the convergence to the trajectory is guaranteed after a transient behavior. It can be observed that the X4 platform tends to the target point precisely, which demonstrates that the proposed approach succeeds in station keeping control for the X4-flyer platform with minimum energy (see Figure 9). Simulation results with the quadrotor helicopter starting with initial condition different from the reference trajectory (see Figure 11).

The results performance of this technique are discussed and compared to the results presented in $[22,23]$. The simulation results showed that the quadrotor UAV can be stabilized at the desired with minimum energy in control effort. PD-CTC in this paper has shown better performance of X4.
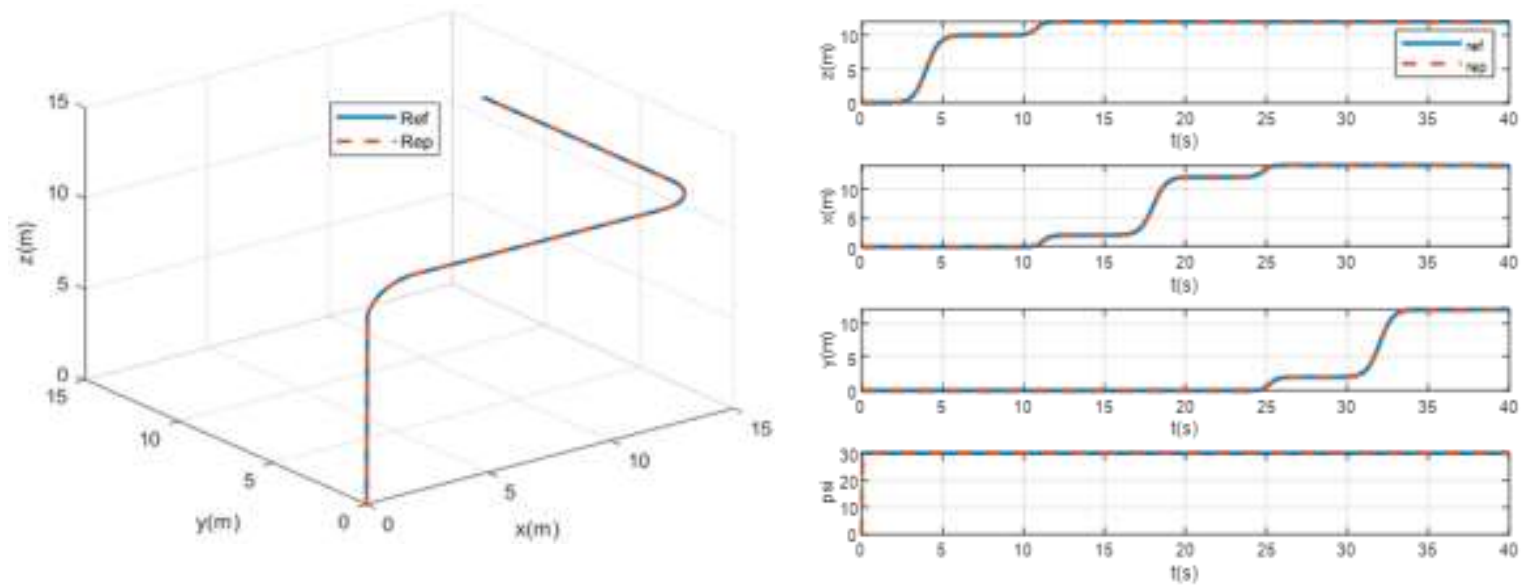

Figure 8. Result of position trajectories 

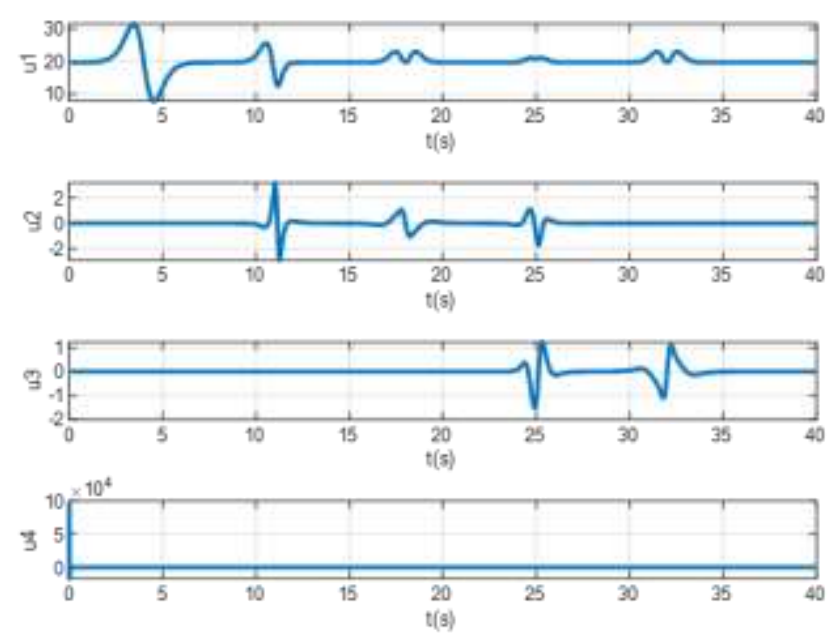

Figure 9. Results of the controllers
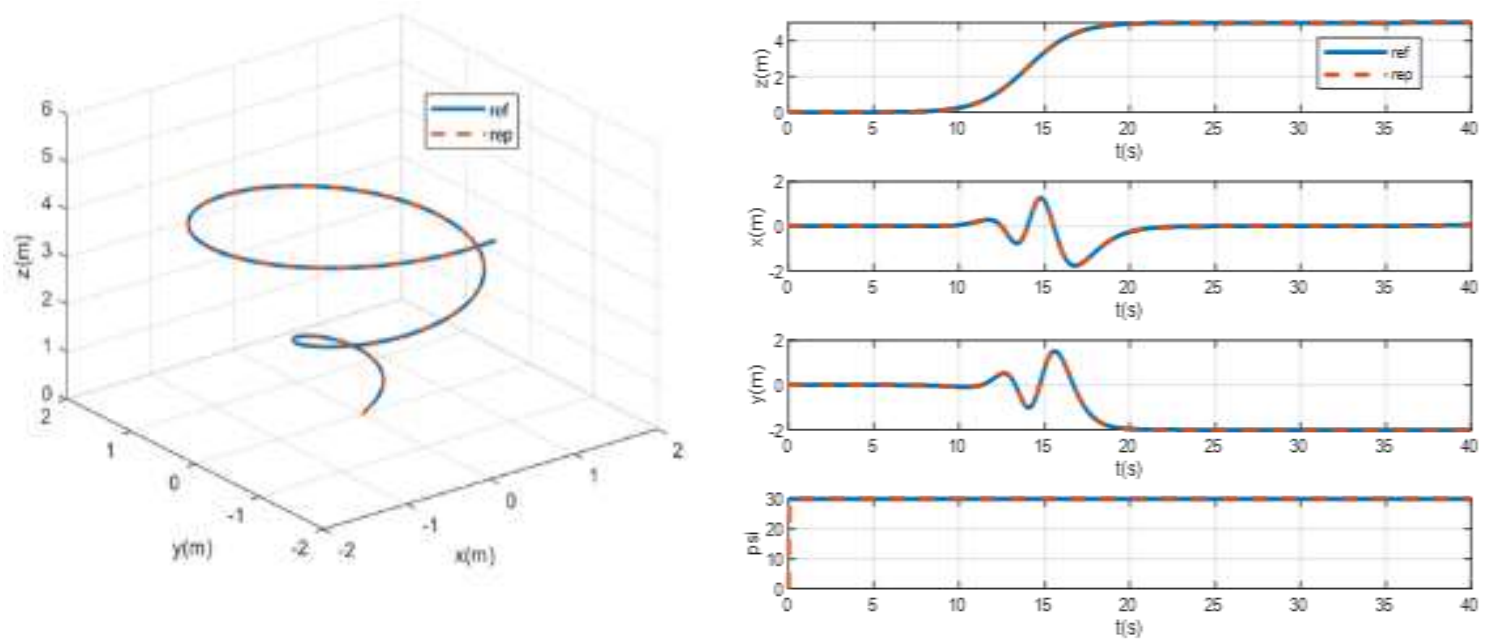

Figure 10. Result of the conical trajectories

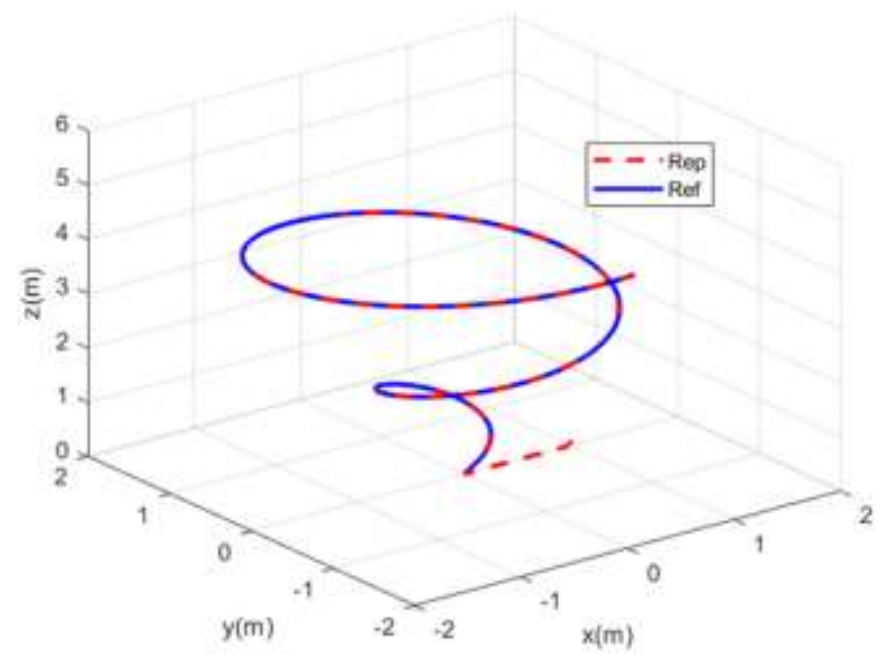

Figure 11. Result of the conical trajectories with initial conditions 


\section{CONCLUSION}

The nonlinear computed torque control for a quadrotor is investigated in this paper. The simulation results demonstrate the X4 can stabilize at the desired with minimum energy in control effort. Further investigations are undergoing on robust controllers that would be able compensate noise effects and parameters mismatch, to be afterwards implemented on a real time experimental setup. Obstacles avoidance and flying multi-drone are also envisaged in a related research work.

\section{REFERENCES}

[1] S. McGilvray, A.Tayebi, "Attitude Stabilization of a VTOL Quadrotor Aircraft," IEEE Trans on control systems technology, Vol.14, pp.562-571, 2006

[2] S. Bouabdallah, R. Siegwart, "Backstepping and Sliding mode Techniques Applied to an Indoor Micro Quadrotor, " In Proc. of the IEEE Inter Conf on Robotics and Automation, pp. 2247 2252, 2005.

[3] L. Beji K. M. Zemalache and H. Marref," Control of an under- actuated system: Application to a four rotors rotorcraft," IEEE Inter Conf on Robotics and Biomimetics, pp. 404 - 409, 2005.

[4] P. Castillo, A. Dzul, R. Lozano," Real-time stabilization and tracking of a four-rotor mini rotorcraft". IEEE Trans. On Control Systems Technology, Vol.12, No. 4, July 2004, pp. 510 - 516, 2004.

[5] H. Voos. "Nonlinear Control of a Quadrotor Micro-UAV using Feedback-Linearization," In Proc. of the IEEE Inter Conf on Mechatronics (ICM 2009), Malaga, Spain, 2009.

[6] P. McKerrow, "Modeling the Draganflyer for-rotor helicopter", Proceedings of the 2004 IEEE Inter. Conf. on Rob. \& Automation, New Orleans, LA, 2004, pp. 3596-3601.

[7] S. Bouabdallah, P. Murrieri, and R. Siegwart, "Design and Control of an Indoor Micro Quadrotor", Proceedings of the 2004 IEEE International Conference on Robotics \& Automation, New Orleans, LA, 2004, pp. 4393-4398.

[8] H. Bouadi, M. Bouchoucha and M. Tadjine, "Sliding Mode Control based on Backstepping Approach for an UAV Type-Quadrotor”, International Journal of Applied Mathematics and Computer Sciences, Vol. 4 No. 1, 2008.

[9] J. Colorado, A. Barrientos, A. Martinez, B. Lafaverges, and J. Valente, "Mini-quadrotor Attitude Control based on Hybrid Backstepping and Frenet-Serret Theory”, IEEE, International Conference on Robotics and Automation (ICRA), 2010.

[10] Zachary T. Dydek, Anuradha M. Annaswamy and Eugene Lavretsky, “Adaptive Control of Quadrotor UAVs in the Presence of Actuator Uncertainties”, American Institute of Aeronautics and Astronautics (AIAA), Atlanta, Georgia, April 2010.

[11] Zhou Fang, Zhang Zhi, Liang Jun and Wang Jian, "Feedback Linearization and Continuous Sliding Mode Control for a Quadrotor UAV', Proceedings of the 27th Chinese Control Conference July, Kunming, Yunnan, China, 2008.

[12] DongBin Lee, Timothy C. Burg, Darren M. Dawson, Dule Shu, Bin Xian and Enver Tatlicioglu, "Robust Tracking Control of an Underactuated Quadrotor Aerial-Robot Based on a Parametric Uncertain Model", Clemson University CRB, Technical report, 2009.

[13] Daewon Lee, H. Jin Kim, and Shankar Sastry, "Feedback Linearization vs. Adaptive Sliding Mode Control for a Quadrotor Helicopter", International Journal of Control, Automation, and Systems, Vol. 7, N. 3, pp 419-428, 2009.

[14] Alaeddin Bani Milhim, Youmin Zhang and Camille-Alain Rabbath, "Gain Scheduling Based PID Controller for Fault Tolerant Control of a Quad- Rotor UAV”, American Institute of Aeronautics and Astronautics (AIAA), Atlanta, Georgia, 20-22 April 2010.

[15] Alexis K. Nikolakopoulos, G. Tzes, "Constrained optimal attitude control of a quadrotor helicopter subject to wind-gusts: Experimental studies”, American Control Conference (ACC), 2010, pp 4451-4455.

[16] Guilherme V. Raffo, Manuel G. Ortegaa and Francisco R. Rubio, “An integral predictive/nonlinear $H_{\infty}$ control structure for a quadrotor helicopter”, Automatica, Vol. 46, N. 1, January 2010, pp 29-39.

[17] K. Zemalache Meguenni, Abdelhamid Drif, M.Tahar, Ryadh Hadj Mokhneche, L. Mostefai and M. R. Benhadria "Fuzzy Integral Sliding Mode Controller for an Autonomous Helicopter", Journal of Cybernetics and Informatics (JCI), pp. 13-27, Vol. 13, 2012.

[18] K. Zemalache Meguenni, M. Tahar, M. Benhadria and Y. Bestaoui, "Integral Backstepping Controller for an Autonomous Helicopter", International Review of Aerospace Engineering (IREASE), Vol. 3, N. 5, October 2010, pp. 257-267.

[19] Zongyu Zuo, Ming Zhu and Zewei Zheng, "Trajectory Tracking Control of a Quadrotor Unmanned MiniHelicopter", 48th AIAA Aerospace Sciences Meeting Including the New Horizons Forum and Aerospace Exposition, Orlando, Florida, January 2010.

[20] K. M. Zemalache, L. Beji and H. Maaref, "Two Inertial Models of X4-Flyers Dynamics, Motion Planning and Control”, Journal of the Integrated Computer-Aided Engineering (ICAE), Vol. 14, N. 2, January 2007, PP. 107119.

[21] K. M. Zemalache and H. Maaref, "Controlling a drone: Comparison between a based model method and a fuzzy inference system", Journal of Applied Soft Computing, Vol.9, N 2, 2009, pp. 553-562.

[22] Noordin1, M. A. M. Basri2, Z. Mohamed, "Sliding mode control for altitude and attitude stabilization of quadrotor UAV with external disturbance”, IJEEI, Vol. 7, No. 2, June 2019, pp. 203 210.

[23] Yuan Chen , Kangling Wang and Wei Chen, "Adaptive fuzzy depth control with trajectory feedforward compensator for autonomous underwater vehicles", Advances in Mechanical Engineering 2019, Vol. 11(3) 1-12 
[24] H. S. Khan and M. B. Kadri, "Attitude and altitude control of quadrotor by discrete PID control and non-linear model predictive control," 2015 Int. Conf. Inf. Commun. Technol., pp. 1-11, 2015.

[25] S.L. Waslander, G.M. Hoffmann, S.J. Jung, and C.J. Tomlin, ”Multi-agent quadrotor testbed control design: integral sliding mode vs reinforcement learning," IEEE/RSJ Inter Conf on Intelligent Robots and Systems (IROS 2005), 2005.

[26] T. I. Fossen, Guidance and Control of Ocean Vehicles John Wiley Sons Ltd, Chichester, (1994).

\section{BIOGRAPHIES OF AUTHORS}

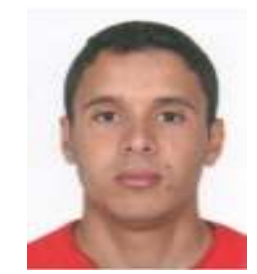

Meddahi Youssouf was born in 1986, received the Master Degree in Electrical Engineering from University of Sciences and Technology of Oran M-B (USTO), Algeria, in 2009 and received the $\mathrm{PhD}$ from USTO-MB, in 2015. His research interests include nonlinear control of mechanical systems, robotics, and control system analysis and design tools for under-actuated systems with applications to aerospace vehicles.

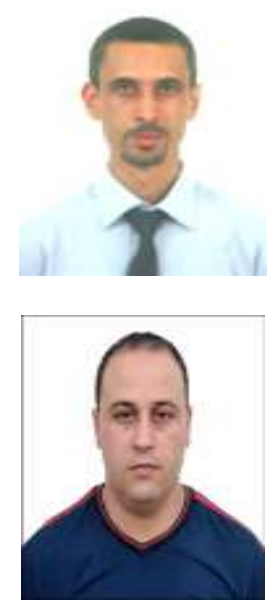

Kadda Zemalache Meguenni received the engineering Degree in Electrical Engineering from University of Sciences and Technology of Oran M-B (USTO), Algeria, in 1998, Master Degree from USTO in 2001 and received the PhD from Evry University, France, in 2006. His research interests include nonlinear control of mechanical systems, robotics, and control system analysis and design tools for under-actuated systems with applications to aerospace vehicles in LDEE laboratory.

Aoued Houari Was born on 1985 in Relizane, Algeria. He received his B.S (State engineer) Engineering degree in automatism from University of Sciences and Technology of Oran (USTOMB), Algeria, in 2008. He received his M.S (Magister) degree in Electronics, Automatic, Robotic and productic option from USTO-MB university in 2012.He is currently an associate professor at UHBCuniversity.

His main research interest is within the control and applications of advanced control technics, Design and control of UAV. 